\title{
Recurrent Cholestatic Jaundice in Lean Nonalcoholic Steato-Hepatitis in a 27 Year Male
}

\author{
Ajay Vardaan, Amar Deep \\ Department of Medical Gastroenterology, King George's Medical University, Lucknow, India
}

Email address:

jsa.amardeep@gmail.com (A. Deep),drajaymd12345@gmail.com (A. Vardaan)

To cite this article:

Ajay Vardaan, Amar Deep. Recurrent Cholestatic Jaundice in Lean Nonalcoholic Steato-Hepatitis in a 27 Year Male. International Journal of Nutrition and Food Sciences. Vol. 4, No. 4, 2015, pp. 483-185. doi: 10.11648/j.ijnfs.20150404.20

\begin{abstract}
Recurrent cholestasis is not described as a feature of nonalcoholic steato-hepatitis (NASH). We describe a lean patient with recurrent jaundice and pruritus whose liver biopsy showed feature of NASH. His laboratory work up failed to establish any other etiology. His symptoms proved on balanced nutrition.
\end{abstract}

Keywords: Cholestatic Jaundice, NASH, NAFLD

\section{Background}

Nonalcoholic fatty liver disease (NAFLD) is a clinicopathologic syndrome that encompasses a broad spectrum ranging from simple steatosis to nonalcoholic steato-hepatitis (NASH), which may be advance to cirrhosis and end-stage liver disease. From the EASL Clinical Practice Guidelines: Management of cholestatic liver diseases Cholestasis is an impairment of bile formation and/or bile flow which may clinically present with fatigue, pruritus and, in its most overt form, jaundice[7]. The prevalence of NAFLD average $20 \%$ and that of NASH, $2 \%$ to $3 \%$ [1]. In the other literature it is shown that Non-alcoholic fatty liver disease (NAFLD) is now the commonest cause of chronic liver disease, affecting up to $30 \%$ of the general population and $70-90 \%$ of high-risk individuals [8-11]. One of risk factor may be oxidative stress which is considered to play a central role in the pathogenesis of NAFLD because the increased production of ROS is known to cause lipid peroxidation, followed by activation of the inflammatory response, and of stellate cells, leading to fibrogenesis [14]. Lipid peroxidation usually leads to the formation of peroxyl radicals, which are the central species of the peroxidation chain reaction [15]. That's why ROS may be one of the causes of Lean NASH. Nonalcoholic fatty liver disease (NAFLD) has become the most common cause of chronic liver disease (CLD) in the United States and others developed countries. Nonalcoholic fatty liver disease and nonalcoholic steato-hepatitis (NASH) in the absence of overweight and obesity, defined by the anthropometric parameter body mass index (BMI) has been designated "Lean NASH"[1]. NAFLD is mostly asymptomatic. Patients with NASH have an increased risk of progression to cirrhosis, liver failure and hepatocellular carcinoma, [12] and are expected to become the commonest indication for liver transplantation in forthcoming years [13]. Recurrent cholestasis is not described in literature as a feature of NASH NASH is associated with nutritional abnormalities such as obesity, rapid weight loss and total parenteral nutrition. Malnutrition, regardless of its cause, can lead to liver damage and impaired liver function [2]. For example, children in underdeveloped countries whose diets do not contain enough protein can develop a disease called kwashiorkor. One symptom of this disorder is the accumulation of fat in the liver, a condition known as fatty liver [2].

\section{Case Presentation}

A 27 year old male, computer teacher presented to us with history of recurrent, self-resolving jaundice with pruritus for last seven years. There were six episodes, each lasting one and half to two months and has no history suggestive of decompensated liver disease. There was no history of hepatotoxin and drug exposure and also there was no family history suggestive of such illness. His dietary habits were quite irregular and imbalanced in terms of nutrients. Examination showed deep icterus. His weight, height and body mass index were $46 \mathrm{~kg} ., 160 \mathrm{~cm}$ and $17.9 \mathrm{~kg} / \mathrm{m} 2$. Rest of the general and systemic examinations was normal. 


\section{Investigations}

His laboratory studies showed haemoglobin-13.1gm\%, total leucocyte count-5800/cumm, platelets counts $1,56,000 / \mathrm{mm} 3$, mean corpuscular volume $-87 \mathrm{fl}$, mean corpuscular haemoglobin-31pg, mean corpuscular haemoglobin concentration-33g/dL, serum creatinine$0.7 \mathrm{mg} / \mathrm{dL}$, serum bilirubin-25.46mg/dL, aspartate transaminases-69.81 IU/L, alanine transaminases-50.90 IU/L, alkaline phosphate $-688.6 \mathrm{IU} / \mathrm{L}$, serum protein $-6.8 \mathrm{~g} / \mathrm{dl}$, serum albumin $3.2 \mathrm{~g} / \mathrm{dl}$ and abdominal ultrasonography showed normal size and shape of liver with hyper echoic texture. Portal venous system was normal in caliber. Magnetic resonance cholangio pancreatography (MRCP) was normal. HBsAg, anti HCV and HIV were nonreactive. Serum ferritin was $128 \mu \mathrm{g} / \mathrm{L}$ and ceruloplasmin $88 \mathrm{mg} / \mathrm{dL}$. Previous laboratory reports were similar. Antinuclear antibodies, antismooth muscle antibody and anti-mitochondrial antibodies were negative. Liver biopsy [Figure: 1] showed largely maintained liver architecture with prominent periportal hepatocyte ballooning (Brunt Score-2) [6]. There was portal and periportal mixed inflammation. Intracanalicular and cellular cholestasis was seen with hepatic rosette formation. Steatosis was present but was involving $<33 \%$ of liver parenchyma (Brunt Score-1). Spotty necrosis was also evident $<2$ foci X 200 field (Brunt Score-1). Fibrosis was present but was only portal and periportal (Brunt Score-1). However glycogenization of nuclei and Mallory hyaline was not evident. In view of negative serology and autoimmune markers most probable diagnosis was NASH with cholestasis.

\section{Treatment}

Patient was managed with balanced diet, symptomatic treatment for pruritus and oral multivitamins including vitamin E 1000 IU daily.

\section{Outcome and Follow up}

His pruritus gradually subsided in two weeks and jaundice in six weeks. He is being followed on balanced diet and life style.

\section{Discussion}

Lean NASH is an exception in terms of relationship with BMI even though most of pathophysiological changes characterize metabolic syndrome and insulin resistance are present. Initially lean NASH was described in Asians. Asians Indian males were shown to have low insulin sensitivity and higher hepatic trigycerides [1]. Clinical presentations are similar in both Lean and obese NASH. Our case was unusual as he presented as recurrent cholestatic jaundice. This pattern is not described in literature. Malnourished individual ingest less than the recommended daily amounts of carbohydrates, proteins, fats, vitamins and minerals, resulting in serious disorders. Proteins are essential components of all cells including transport channels of hepatocytes and cholangiocytes. An abnormality in the normal balance of various types of amino acids due to malnutrition is expected to increase the risk of cholestasis due to transport channel dysfunction. There is no proven effective therapy for the lean patient with NASH or for the obese patient who fails to improve with weight reduction or simply cannot lose substantial weight [2]. His jaundice improved with balanced nutrition and exercise. So we can say lean NASH may improve by balance diet and balanced life style. Even enough histological cholestasis and clinical jaundice may be present in all stages of alcoholic liver disease [3, 4]. The clinical and histological picture of NASH does not include any references to possible cholestatic coarse due to bile duct involvement. Certainly during prolonged cholestasis, exposure of hepatocyte to toxic bile acids (i.e. hydrophobic) likely promotes liver damage, cholestasis in cholestatic disease contribute to the progressive histological cirrhosis of liver [5, $6]$.

\section{Learning Points}

- Lean NASH can present as recurrent cholestatic jaundice.

\section{Conclusion}

NASH can be kept as a possibility in case of recurrent cholestatic jaundice in a lean patient although it need further validation.

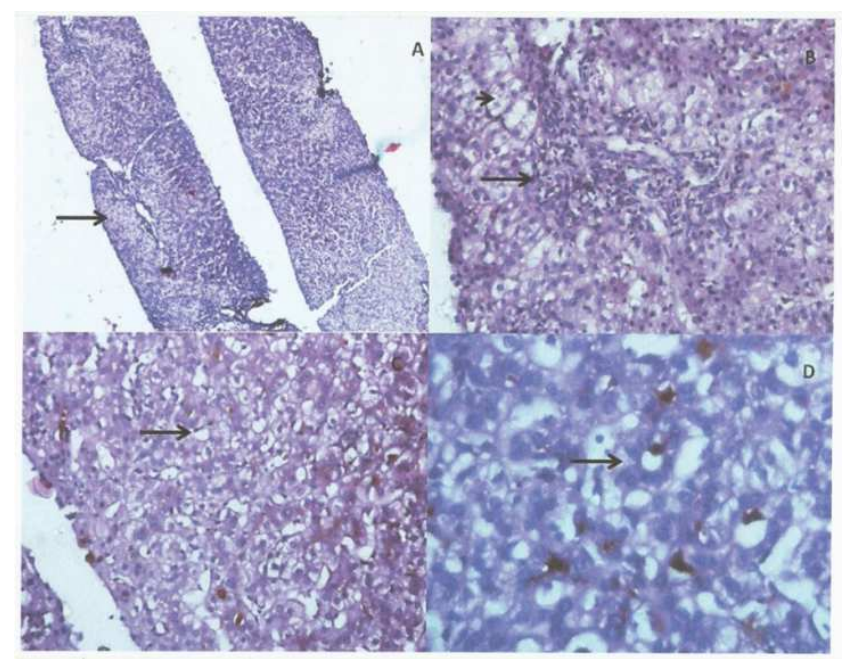

Figure 1. Section shows perisepatal involvement (A) with portal and periportal mixed inflammatory cell infiltrate ( $B$, arrow), macro vesicular and micro vesicular steatosis $(C)$, hepatocyte ballooning (B small arrow) and cholestasis with hepatic rosette formation (D).

\section{Histopathology}

Liver biopsy showed largely maintained liver architecture with prominent periportal hepatocyte ballooning (Score-2) [According to Histological score for NAFLD, Brunt et al]. There was portal and periportal mixed inflammation. 
Intracanalicular and cellular cholestasis was seen with hepatic rosette formation. Steatosis was present but was involving $<33 \%$ of liver parenchyma (Score-1) [Figure: 1-B]. Spotty necrosis was also evident $<2$ foci X 200 field (Score1). Fibrosis was present but was only portal and periportal (Score-1). However glycogenization of nuclei and Mallory hyaline was not evident. In view of negative serology and autoimmune markers most probable diagnosis was NAFLD with cholestasis.

\section{References}

[1] Kausik D, Abhijit C. Lean NASH: distinctiveness and clinical implication. Hepatol Int, 2013, 7 (Suppl 2) S806 S813.

[2] Charls S. Libeber. Relationship between nutrition, alcohol use and liver disease. Alcohol Research and Health. 2003: Vol.27, No.3.

[3] Tung BY, Carithers RL. Cholastasis and alcoholic liver disease. 1999: Clin Liver Dis. 3:585-601.

[4] Afshani P, Littenberg GD, Wollman J, Kaplowitz N. Significance of microscopic cholangitis in alcoholic liver disease. Gastroenterology. 1978; 75:1045-1050.

[5] Lee RG, Alcoholic and Nonalcoholic Steatohepatitis Pathology. In Clinical Pathological Correlation in Liver Disease: Approaching the Next Millennium. Bloom JR, Goodman ZD, IShak KG, (eds). Washington DC, Armed Forces Institute of Pathology, American Registry of Pathology, AASLD, 1998; pp 274-283.

[6] Brunt EM, Janney GG, DiBisceglie AM, Neuschwander-Tetri BA, Bacon BR. Nonalcoholic Steatohepatitis: A Proposal for
Garding and Staging the Histological Lesions. Am J Gastroenterol, 1999; 94:2467-2474 crossRef.

[7] European Association for the Study of the Liver / Journal of Hepatology 51 (2009) 237-267.

[8] Armstrong MJ, Houlihan DD, Bentham L, et al. Presence and severity of non-alcoholic fatty liver disease in a large prospective primary care cohort. J Hepatol 2012; 56:234-40.

[9] Bellentani S, Tiribelli C, Saccoccio G, et al. Prevalence of chronic liver disease in the general population of northern Italy: the Dionysos Study. Hepatology 1994; 20:1442-9.

[10] Browning JD, Szczepaniak LS, Dobbins R, et al. Prevalence of hepatic steatosis in an urban population in the United States: impact of ethnicity. Hepatology 2004;40:1387-95.

[11] Bellentani S, Bedogni G, Miglioli L, et al. The epidemiology of fatty liver. Eur J Gastroenterol Hepatol 2004; 16:1087-93.

[12] Bugianesi E, Leone N, Vanni E, et al. Expanding the natural history of nonalcoholic steatohepatitis: from cryptogenic cirrhosis to hepatocellular carcinoma. Gastroenterology 2002; 123:134-40.

[13] Charlton MR, Burns JM, Pedersen RA, et al. Frequency and outcomes of liver transplantation for nonalcoholic steatohepatitis in the United States. Gastroenterology 2011; 141:1249-53.

[14] Yang SQ, Zhu H, Li Y, Gabrielson K, Trush MA \& Diehl AM: Mitochondrial Adaptations to Obesity-Related Oxidant Stress. Arch Biochem Biophys. 2000; 378(2):259-268.

[15] Torricelli P., Ferorelli P., De Martino A., Antonelli F., Beninati S.. The Influence of Preventive Multiple Micronutrients Supplementation on Liver Steatosis in High-cholesterol Fed C57BL6/N Mice. American Journal of Life Sciences. Vol. 1, No. 2, 2013, pp. 55-60 doi: 10.11648/j.ajls.20130102.16 